\title{
Angular Response of Magnetostrictive Thin Films
}

\author{
Jianjun Li ${ }^{1,2}$ \\ ${ }^{1}$ National Key Laboratory of Science and Technology on Advanced Composites in Special Environments, \\ Harbin Institute of Technology, Harbin 150080, China \\ ${ }^{2}$ Laboratoire de Magnétismde Bretagne, Université de Bretagne Occidentale, 6 Avenue le Gorgeu C.S. 93837, \\ 29238 Brest Cedex 3, France
}

Correspondence should be addressed to Jianjun Li, ljj8081@tom.com

Received 14 November 2011; Revised 12 January 2012; Accepted 13 January 2012

Academic Editor: Ovidiu Crisan

Copyright ( 92012 Jianjun Li. This is an open access article distributed under the Creative Commons Attribution License, which permits unrestricted use, distribution, and reproduction in any medium, provided the original work is properly cited.

The magnetostrictions of the single TbFe layer and coupled $\mathrm{Py} / \mathrm{TbFe}_{2}$ bilayers were measured by using laser deflectometry. The dependences of the magntostriction performance on the driving magnetic field direction have been investigated. The relationship studies between the saturation bending angle and torsion angle of the single layer with perpendicular anisotropy and coupled bilayers with in-plane uniaxial anisotropy have been conducted. Interesting "jump" reflecting the spin dynamics is observed in the magnetostriction loops of the coupled bilayers.

\section{Introduction}

Magnetostrictive films have already shown promising applications in micromechanical devices since the discovery of giant magnetostriction in the Laves phase of $\mathrm{TbFe}_{2}$ by Clark and Belson in 1972 [1, 2]. In the past, extensive experimental studies were undertaken to investigate the relationships between the observed magnetostrictive properties and the film composition, preparation conditions, anisotropy, and so forth, [3-8]. In order to reduce the huge magnetic switching field for $\mathrm{TbFe}_{2}$ alloy and make it suitable for practical applications, Quandt and coworkers combined the rare-earthtransition-metal (RE-TM) alloys with soft magnetic materials, which have high magnetization to form coupled multilayers [9, 10]. The interfacial exchange-coupling between the RE-TM alloys and the soft magnetic materials plays an important role in reducing the magnetic switching field. The magnetostriction of magnetic exchange-spring multilayers has been investigated [11-14], but the first and the last layer are not in the same conditions as the internal layers in a multilayer system. Thus, their properties represent an average of different parameters. Eventual technological differences in the properties of different layers and interfaces are also hidden in a multilayer system. It is necessary and very useful to study the magnetostriction of magnetically coupled bilayers so as to reach better understanding of the mic- roscopic spin configuration in such magnetic thin films. In this paper, the weak magnetostrictive signals of $\mathrm{S} / \mathrm{Py} / \mathrm{TbFe}_{2}-$ coupled bilayers were obtained successfully and the dependences of the saturation magnetostriction of $\mathrm{S} / \mathrm{TbFe}_{2}$-single layer and $\mathrm{S} / \mathrm{Py} / \mathrm{TbFe}_{2}$-coupled bilayers on the driving magnetic field direction were studied.

\section{Experimental}

Rectangular Corning glass was chosen as substrate $(22 \times 5 \times$ $0.16 \mathrm{~mm}^{3}$ ), and $\mathrm{TbFe}_{2}$ and Py layers were deposited onto it from 4 inch targets mosaic by using a Z550 Leybold RF sputtering equipment with a rotary table technique. During the film deposition, a static magnetic field (around $0.03 \mathrm{~T}$ ) was set along the long axis of the rectangular substrate to favour in-plane uniaxial magnetic anisotropy in the soft magnetic film. A $3 \mathrm{~nm}$ copper protective layer was grown onto the samples. The $\mathrm{TbFe}_{2}$ layer is amorphous and sputtered under argon gas pressure of $1.2 \times 10^{-2}$ mbar from an alloy target with a nominal composition of $\mathrm{TbFe}_{2}(99.9 \%$ in purity), while Py is polycrystalline and sputtered under argon gas pressure of $1.2 \times 10^{-2}$ mbar from an alloy target with a nominal composition of $\mathrm{Ni}_{80} \mathrm{Fe}_{20}$ (99.99\% in purity). The deposition rates are $6.2 \AA / \mathrm{s}$ for $\mathrm{TbFe}_{2}$ layer and $4 \AA / \mathrm{s}$ for Py layer. The single layer and coupled bilayers studied in this 


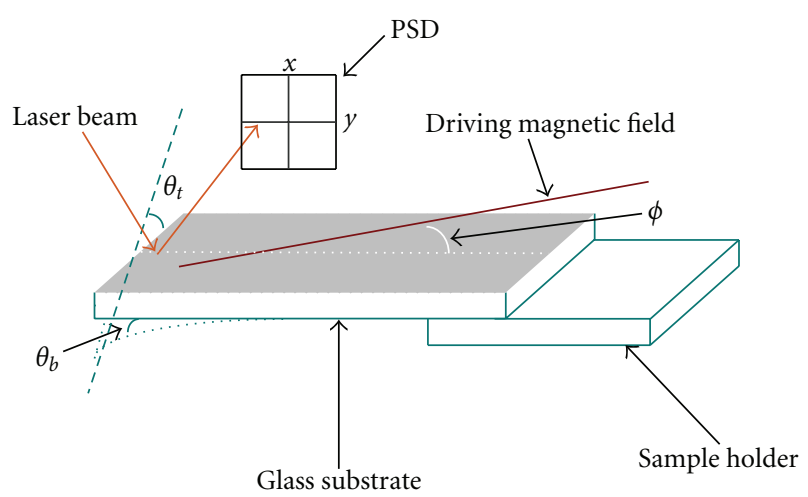

FIGURE 1: Outline of the measurement of magnetoelastic deformations (bending and torsion angles). The dashed line indicates the long axis of the sample. $\phi$ is the angle between the long axis of the sample and the driving magnetic field.

paper had the following configurations: [ $\left.\mathrm{S} / \mathrm{TbFe}_{2}(1.24 \mu \mathrm{m})\right]$ and $\left[\mathrm{S} / \mathrm{Py}(10 \mathrm{~nm}) / \mathrm{TbFe}_{2}(10 \mathrm{~nm})\right]$.

The magnetostrictions of $\mathrm{S} / \mathrm{TbFe}_{2}$-single layer and $\mathrm{S} / \mathrm{Py} / \mathrm{TbFe}_{2}$-coupled bilayers were obtained by laser deflectometry. Figure 1 outlines the magnetostrictive measurement of the magnetic films. He-Ne laser strikes on the sample surface and is reflected by the sample to reach PSD (Hamamatsu S1300 position sensitive detector) surface. When the magnetic field is applied and deformation of the substrate occurs, the deflections can be recorded simultaneously. The PSD gives a voltage proportional to the position of the laser spot on the detecting surface and provides continuous position data on both the $X$ and $Y$ axes, so the angular deformations of the sample (bending angle $\theta_{b}$ and torsion angle $\theta_{t}$ ) can be measured [13]. Angle $\phi$ is the angle between the driving magnetic field and the long axis of the rectangular substrate. In our studies, angle $\phi$ can be varied from 0 to $360^{\circ}$ by rotating the electromagnet. The driving magnetic field is always kept in the film plane. The hysteresis loops were performed on a vibrating sample magnetometer (VSM).

\section{Results and Discussion}

3.1. Single $\mathrm{TbFe}_{2}$ Layer. The magnetostriction loops of the $\mathrm{TbFe}_{2}$-single layer measured at different angles $\phi(\phi=15$, $\left.75,90,105,165,195^{\circ}\right)$ are displayed in Figure 2. When the driving magnetic field is not applied, no magnetostriction occurs. The driving magnetic field up to $0.7 \mathrm{~T}$ is applied for the magnetostriction measurement of $\mathrm{TbFe}_{2}$ single layer. If the magnetic field is not strong enough, the saturation magnetostriction of $\mathrm{TbFe}_{2}$ layer cannot be observed for its the strong anisotropy. In Figure $2(\mathrm{a}), \theta_{b}$ and $\theta_{t}$ reach the maximum negative value at the saturation field, then the absolute values of $\theta_{b}$ and $\theta_{t}$ decrease to zero following the decrease of the driving magnetic field. After the driving magnetic field changes to the opposite direction and starts to increase again, the absolute values of $\theta_{b}$ and $\theta_{t}$ increase smoothly till they reach the same maximum negative values. When the angle $\phi$ is changed by rotating the electromagnet, similar results are observed except the differences of the saturation magnetostriction and the sign of the deformation angle.
Figure 3 shows the saturation magnetostriction of $\mathrm{TbFe}_{2}$ single layer as a function of angle $\phi$. According to the results, $\theta_{\text {bsat }}(\phi)$ and $\theta_{\text {tsat }}(\phi)$ curves have $\cos ^{2}(\phi)$ and $\sin ^{2} \phi$ characteristics, respectively. The functions $\mathrm{A}_{1}\left(\cos ^{2}\left(\phi+\phi_{1}\right)+\mathrm{B}_{1}\right)$ and $\mathrm{A}_{2}\left(\sin ^{2}\left(\phi+\phi_{2}\right)+\mathrm{B}_{2}\right)$ are used to fit, where $\phi_{1}$ and $\phi_{2}$ represent the correction angle of the sample misorientation calculated from bending and torsion angle, and $\mathrm{A}$ and $\mathrm{B}$ are constants: A means the oscillation amplitude and B indicates the easy axis deviation from the long axis of the sample. According to fitting, the simulated curves are shown in Figure 3 and the functions for $\mathrm{TbFe}_{2}$ single layer are $\theta_{\text {bsat }}(\phi)=$ $-1.05\left(\cos ^{2}\left(\phi-2^{\circ}\right)\right) ; \theta_{\text {tsat }}(\phi)=-0.41\left(\sin ^{2}\left(\phi+6^{\circ}\right)+0.24\right)$. According to the equation in [13]

$$
\theta_{\text {sat }}=\frac{6\left(1+v_{s}\right) L t_{f}}{E_{s} t_{s}^{2}} b^{\gamma, 2}
$$

one can calculate the magnetoelastic coupling coefficient $b^{\gamma, 2}$ of the magnetostrictive thin films. Here $E_{s}$ and $v_{s}$ are the Young modulus and Poisson ratio of the substrate, respectively. $L$ is the sample length $(20 \mathrm{~mm})$, while $t_{s}$ represents the glass substrate thickness and $t_{f}$ is the film thickness (including Py and $\mathrm{TbFe}_{2}$ for coupled bilayers). For the glass substrate, $t_{s}$ is $0.16 \mathrm{~mm}$ and $E_{s}=60 \mathrm{GPa}$ and $v_{s}=0.27$. Based on the results, magnetoelastic coupling coefficient of $\mathrm{TbFe}_{2}-$ single layer in our experiments is $b^{\gamma, 2}$ single layer $=8.53 \mathrm{MPa}$, which is similar to what have been reported before [15].

It is known that the $\mathrm{TbFe}_{2}$ film has a perpendicular easy axis. Several models including pair ordering [16], anelastic distortion of magnetic atom environment [17], and hexagonal planar units with a preferred axis perpendicular to the film plane $[18,19]$ have been proposed to account for the origin of the perpendicular anisotropy of $\mathrm{TbFe}_{2}$ film. In ideal ferrimagnetic $\mathrm{TbFe}_{2}$-single layer, the model in Figure 4(a) can be used to explain the angular response of the saturation magnetostriction. Hysteresis loops (Figure $4(\mathrm{~b})$ ) of $\mathrm{TbFe}_{2}$ single layer with field in plane $\left(\phi=0\right.$ and $\left.90^{\circ}\right)$ have no difference. Also the hysteresis loops (Figure 4(c)) of $\mathrm{TbFe}_{2}$ single layer with field in plane and perpendicular to plane confirm the existence of the perpendicular anisotropy in the film. When the magnetic field is applied, the magnetic vectors rotate into the film plane and subsequently change the internal 


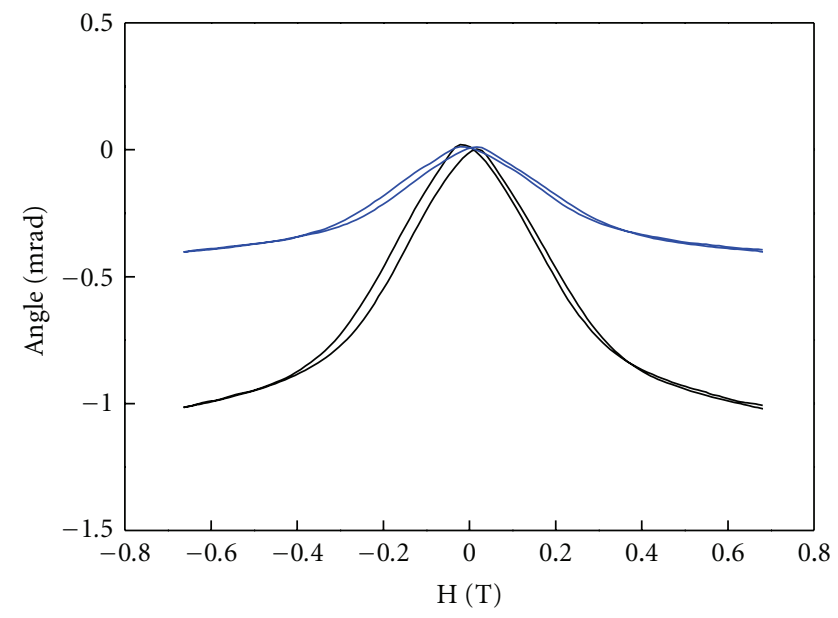

(a)

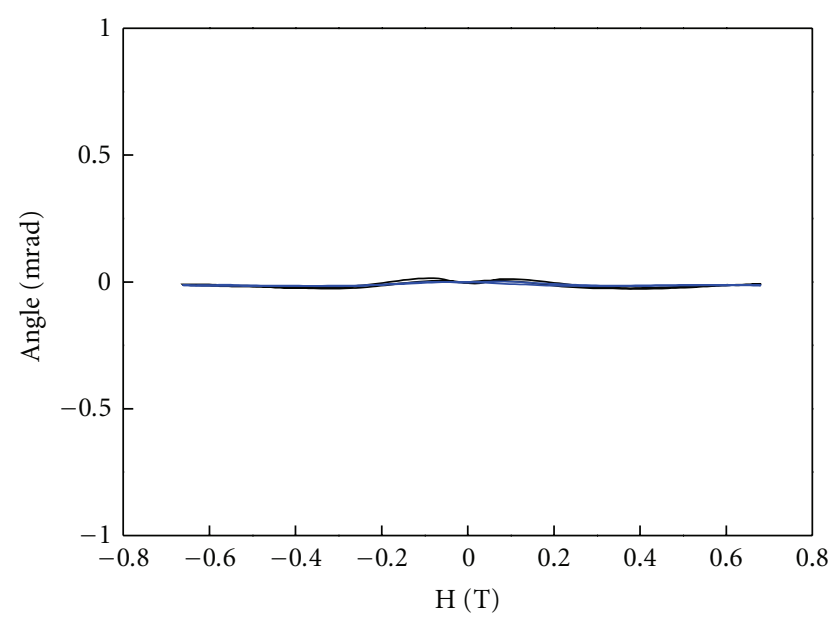

(c)

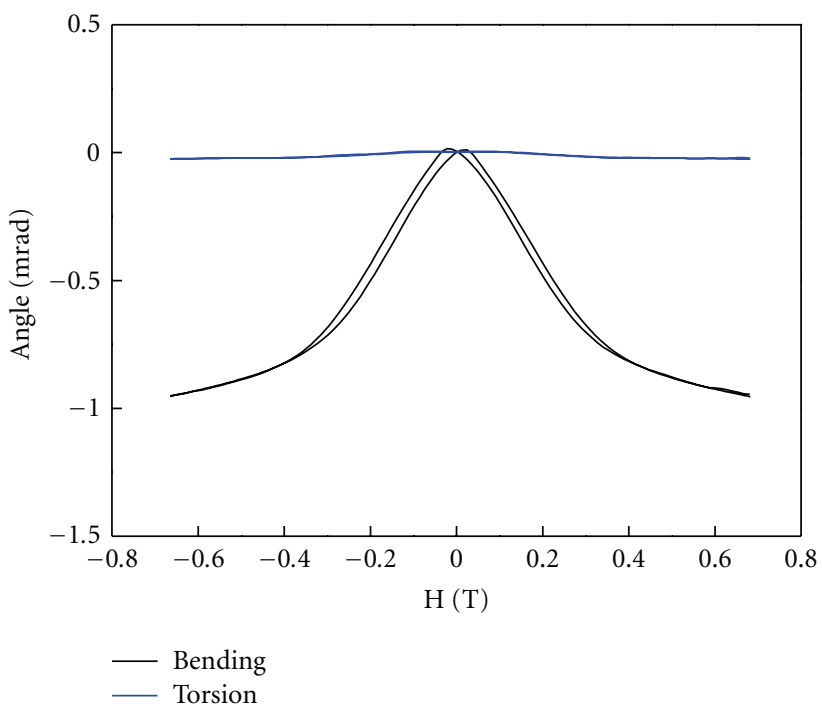

(e)

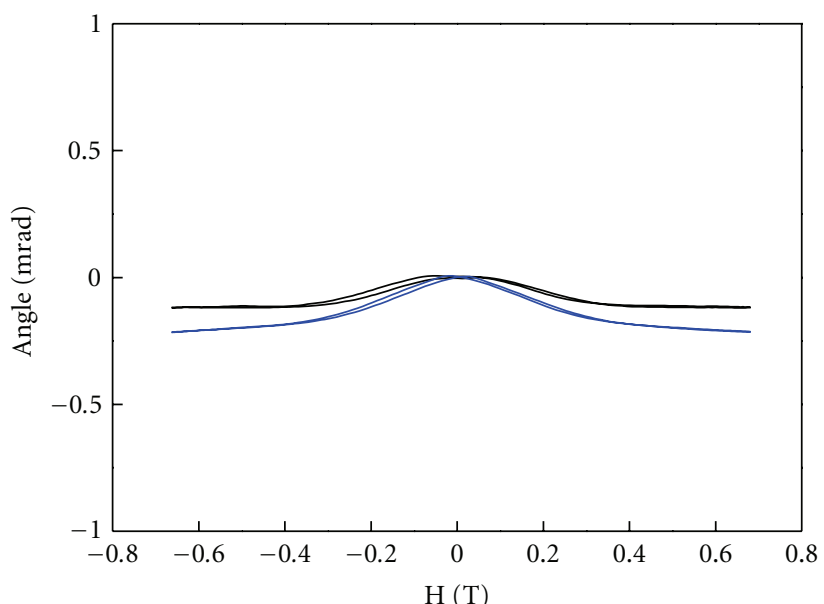

(b)

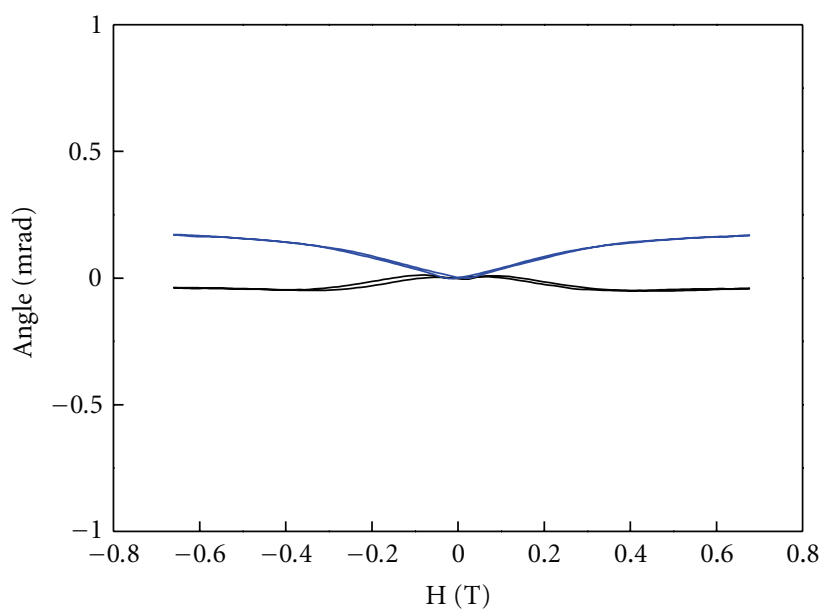

(d)

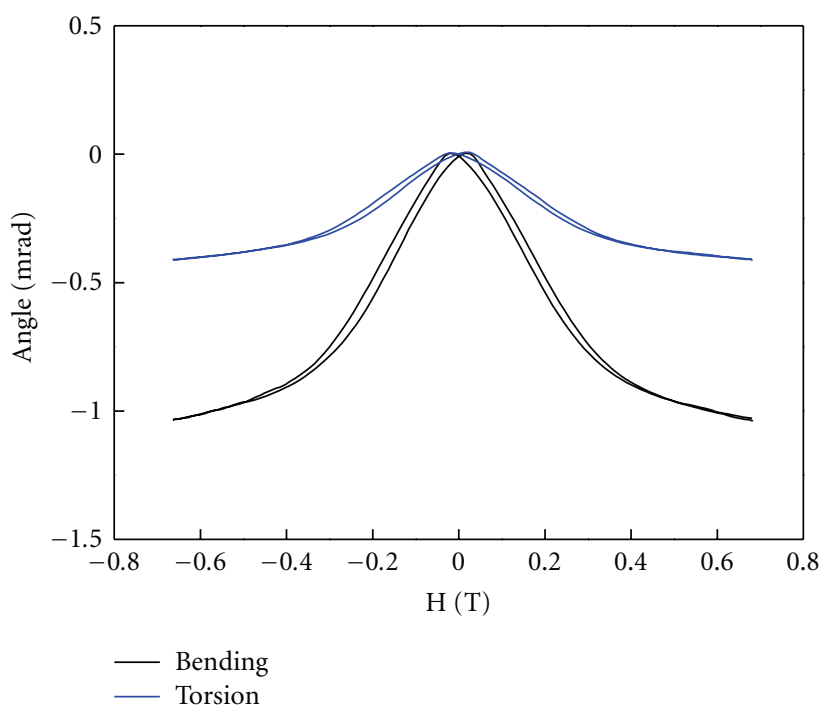

(f)

FIGURE 2: Experimental magnetostriction loops of $\mathrm{TbFe}_{2}$ single layer at different driving field angles $\left(\phi=15,75,90,105,165,195^{\circ}:(\mathrm{a})-(\mathrm{f})\right)$. 


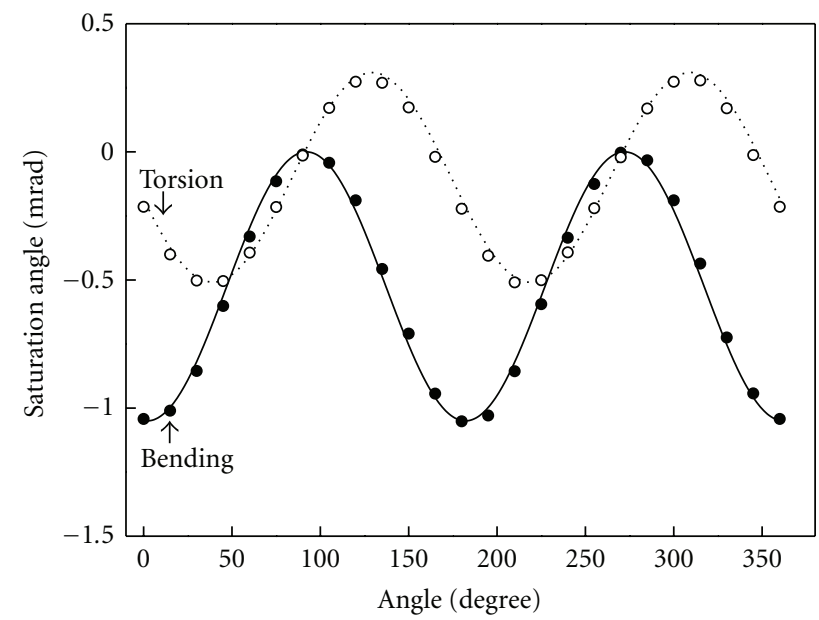

Figure 3: The saturation bending angle $\theta_{b}$ and torsion angle $\theta_{t}$ for $\mathrm{TbFe}_{2}$ single layer as a function of angle $\phi\left(\phi\right.$ varying every $15^{\circ}$ from 0 to $\left.360^{\circ}\right)$. The experimental data are represented by circles, and the fit is illustrated by line (bending: solid; torsion: dot).
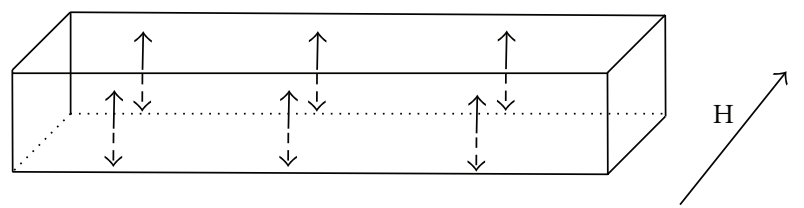

Driving field $\mathrm{H}$ in the film plane

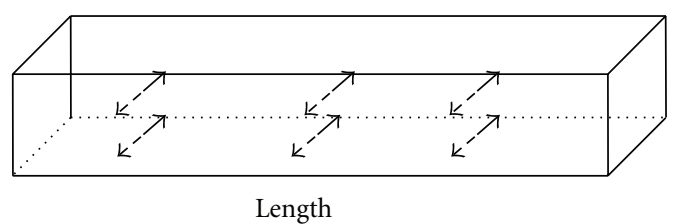

$\longleftrightarrow-\mathrm{TbFe}_{2}$ magnetic vector

(a)

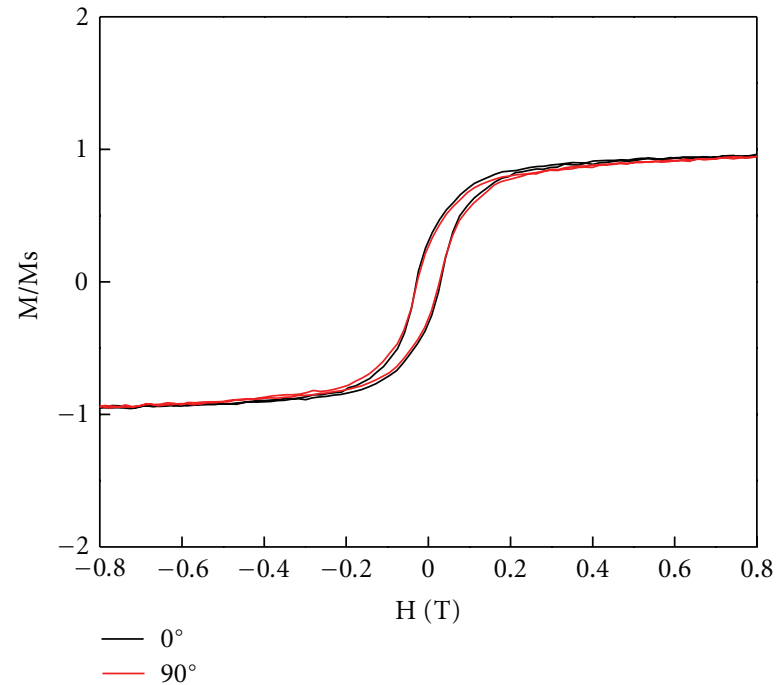

(b)

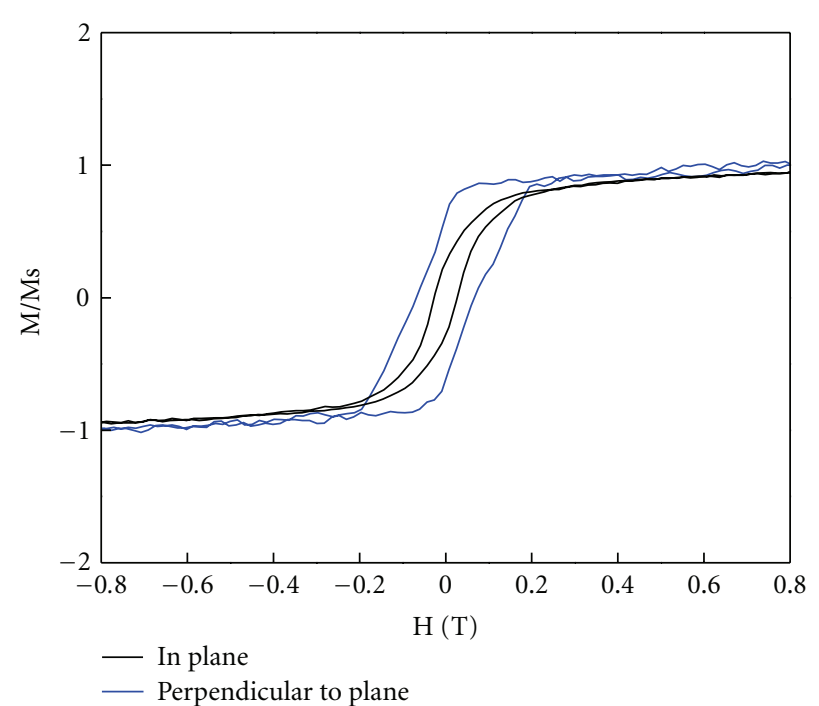

(c)

Figure 4: (a) Schematic drawing of the magnetic vector rotation model for $\mathrm{TbFe}_{2}$ single layer (H: the driving magnetic field); (b) hysteresis loops of single $\mathrm{TbFe}_{2}$ layer with field in plane $\left(\phi=0\right.$ and $\left.90^{\circ}\right)$; (c) hysteresis loops of single $\mathrm{TbFe}_{2}$ layer with field in plane and perpendicular to plane.

stress. The different saturation magnetostrictions are caused by the different $\mathrm{TbFe}_{2}$ spin configurations with varied field direction. The $\mathrm{TbFe}_{2}$ film has the maximum saturation magnetostriction when the $\mathrm{TbFe}_{2}$ vectors rotate into the film plane along the long axis of the sample, but nearly zero magnetostriction is observed when the magnetic field is applied perpendicular to the long axis of the sample $\left(\phi=90^{\circ}\right)$. Such phenomenon results from the rectangular shape of the 


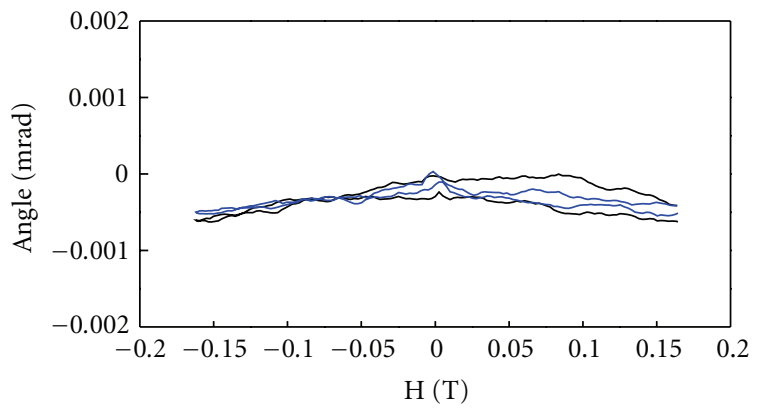

(a)

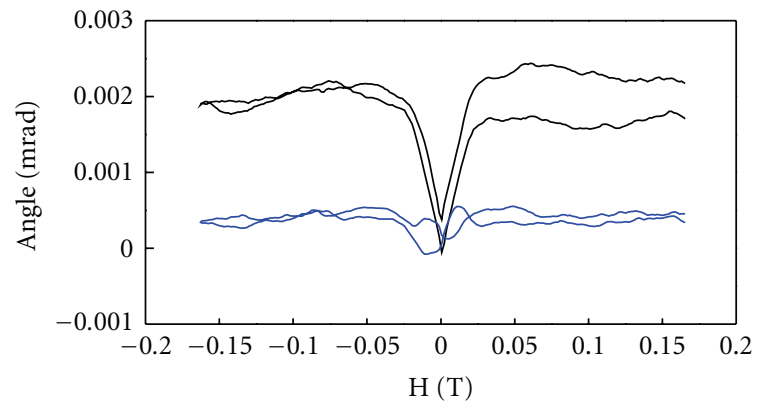

(c)

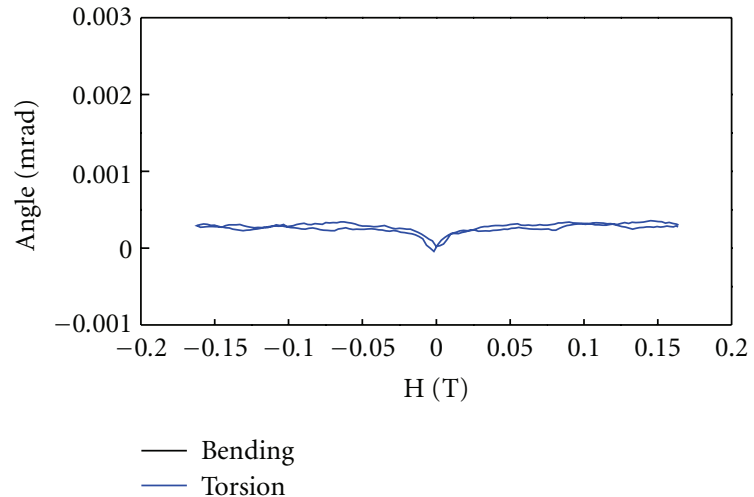

(e)

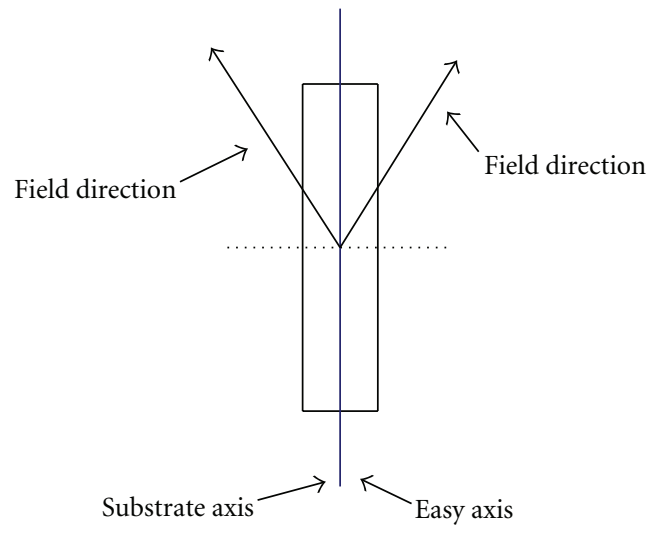

(i)

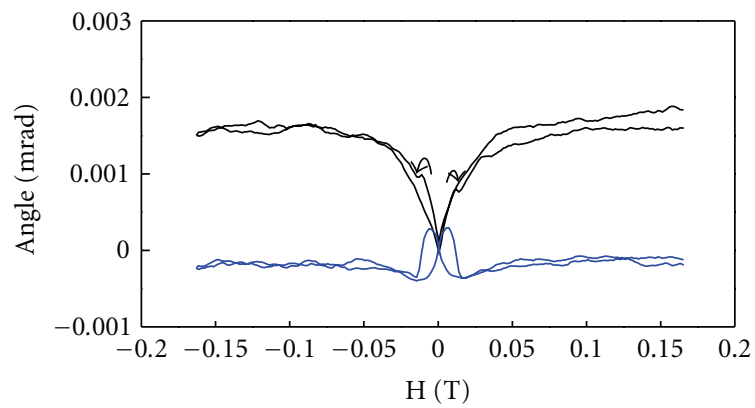

(b)

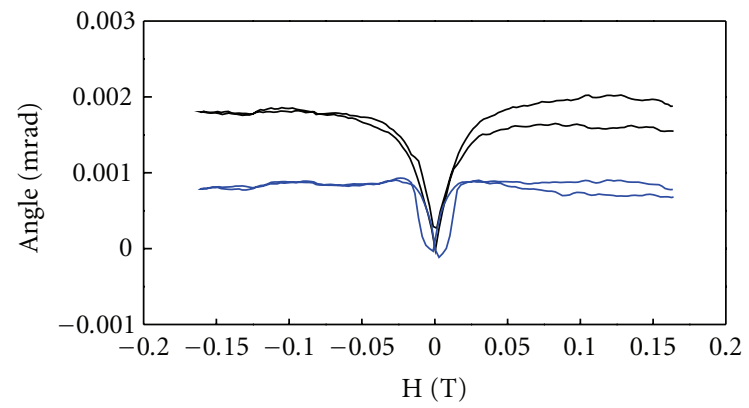

(d)

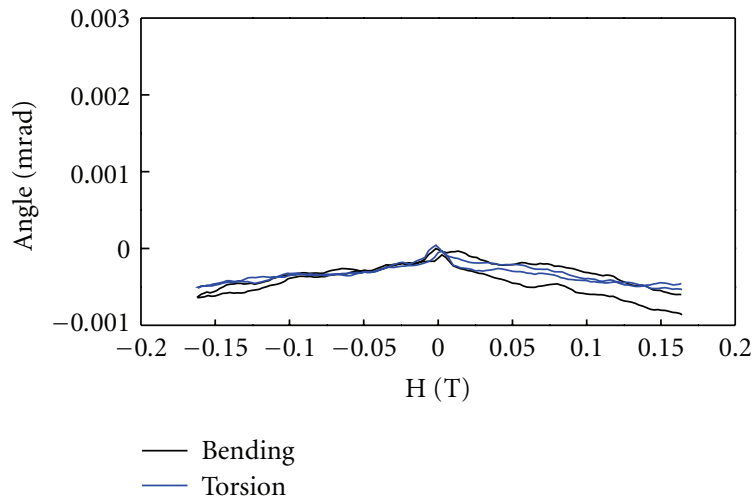

(f)

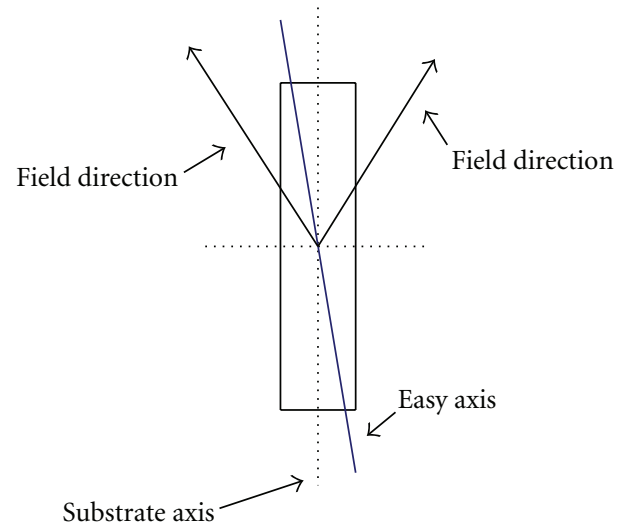

(ii)

(g)

FigURE 5: Representative magnetostriction loops (solid line: bending; dot line: torsion) of $\mathrm{S} / \mathrm{Py} / \mathrm{TbFe}_{2}$-coupled bilayers at different driving field angle $\left(\phi=15,75,90,105,165,195^{\circ}\right.$ : (a)-(f)); graphical representations (i) and (ii) reveal the deviation of easy axis from the long axis of the sample. 


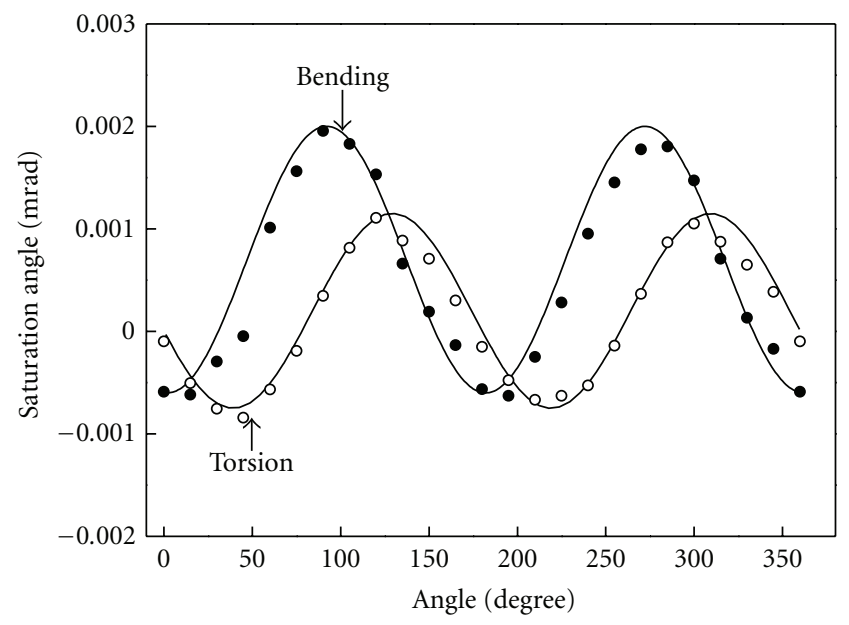

Figure 6: The saturation bending angle $\theta_{b}$ and torsion angle $\theta_{t}$ of $\mathrm{S} / \mathrm{Py} / \mathrm{TbFe}_{2}$ bilayers as a function of angle $\phi\left(\phi\right.$ varying every $15^{\circ}$ from 0 to $\left.360^{\circ}\right)$. The experimental data are represented by circles, and the fit is illustrated by line (bending: solid; torsion: dot).

substrate, and the cantilever structure makes the substrate hard to distort along the perpendicular direction (The cantilever is fixature).

3.2. $\mathrm{S} / \mathrm{P} y / \mathrm{TbFe}_{2}$-Coupled Bilayers. The angular responses of $\mathrm{H}_{\mathrm{EX}}$ (exchange bias) and $\mathrm{H}_{C}$ (coercivity) in FM/AFM coupled bilayers have been reviewed by Ambrose et al. [20]. The angular response of the saturation magnetostriction of $\mathrm{S} / \mathrm{Py} / \mathrm{TbFe}_{2}$-coupled bilayers is investigated in this section. The angle $\phi$ is changed every $15^{\circ}$ from 0 to $360^{\circ}$, and representative magnetostriction loops of the coupled bilayers measured at different angle $\phi\left(\phi=15,75,90,105,165,195^{\circ}\right)$ are shown in Figure 5. In Figure 5(a), small negative bending and torsion occur. When $\phi$ increases, the exchange-coupling effect starts to show more clearly, the saturation torsion angle $\theta_{\text {tsat }}$ in Figure 5(b) is negative, but the saturation bending angle $\theta_{\text {bsat }}$ becomes positive. The "jump" appears in the magnetostriction loop as indicated in Figure 5(b). In the magnetostriction loops of the coupled bilayers, the "jump" is only observed over a restricted range of angle $\phi$. In Figures 5(c) and $5(\mathrm{~d})$, both the saturation bending and torsion angles turn positive. And the magnetostriction loops in Figure 5(f) are the same as that shown in Figure 5(a), implying that $\operatorname{MS}(\phi)$ magnetostriction loop is the same as $\operatorname{MS}\left(\phi+180^{\circ}\right)$. $\operatorname{MS}(\phi)$ and $\operatorname{MS}(-\phi)$ magnetostriction loops of the coupled bilayers are different in our results, which are caused by the deviation of easy axis from the long axis of the sample (graphical representations in Figure 5). A much smaller magnetic field can reach the saturation magnetostriction for the coupled bilayers and the exchange-coupling effect can reduce the switching magnetic field effectively.

The coupled bilayers have a uniaxial anisotropy in the film plane and the maximum saturation magnetostriction appears when $\phi$ is about $90^{\circ}$, which differs greatly from the $\mathrm{TbFe}_{2}$ single layer. The difference comes from the exchangecoupling effect between the $\mathrm{Py}$ and $\mathrm{TbFe}_{2}$ layers, which results in the uniaxial magnetic anisotropy in the film plane. When the field is applied along the easy axis, the magnetization changes by the $180^{\circ}$ domain wall displacement and there is no magnetostrictive effect. To the contrary, if the field is applied along the hard axis, the magnetic vectors turn by $90^{\circ}$ and the maximum saturation magnetostriction is observed. The fitting curves for the saturation magnetostriction of the coupled bilayers shown in Figure 6 also have $\cos ^{2}(\phi)$ and $\sin ^{2} \phi$ behaviours. The functions obtained from the fitting for $\mathrm{S} / \mathrm{Py} / \mathrm{TbFe}_{2}$ coupled bilayers are $\theta_{\mathrm{bsat}}(\phi)=$ $-0.0026\left(\cos ^{2}\left(\phi-2^{\circ}\right)-0.77\right)$ and $\theta_{\text {tsat }}(\phi)=-0.00095$ $\left(\sin ^{2}\left(\phi+6^{\circ}\right)-0.21\right)$. Finally the magnetoelastic coupling coefficient of the coupled bilayers is calculated: $b^{\gamma, 2}$ bilayers $=$ $0.83 \mathrm{MPa}$.

Stoner-Wohlfarth model can be used to explain the magnetostriction results of the coupled bilayers. In the coupled bilayers, Py and $\mathrm{TbFe}_{2}$ magnetic vectors rotate together when driving magnetic field is applied [13]. The rotation of the magnetic vectors will lead to the internal stress change (magnetostrictive stress) in the film, and then magnetostriction occurs (as shown in Figure 7). The "jump" in some magnetostriction loops is caused by the magnetic vectors aligning along the hard axis of the sample, and it reveals the spin dynamics in the process of magnetic vectors rotating from the easy axis to the magnetic field direction. The "jump" will be affected by the driving magnetic field direction and the deviation of easy axis from the long axis of the sample. Further work will be undertaken to explain these features with a model that is more appropriate than Stoner-Wohlfarth model.

\section{Conclusion}

In summary, the magnetostrictions of $\mathrm{S} / \mathrm{TbFe}_{2}$-single layer and $\mathrm{S} / \mathrm{Py} / \mathrm{TbFe}_{2}$-coupled bilayers were measured by using laser deflectometry. The dependences of the magnetostriction on the driving magnetic field direction have been established. Interesting "jump" reflecting the spin dynamics is observed in the magnetostriction loops of the coupled bilayers. These results show that magnetostriction measurement is a very promising technique to reveal the spin configuration in the thin magnetic films. 


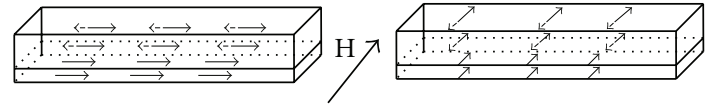

Driving field $\mathrm{H}$ in the film plane

$\longleftrightarrow \mathrm{TbFe}_{2}$ magnetic vector

$\longrightarrow$ Py magnetic vector

(a)

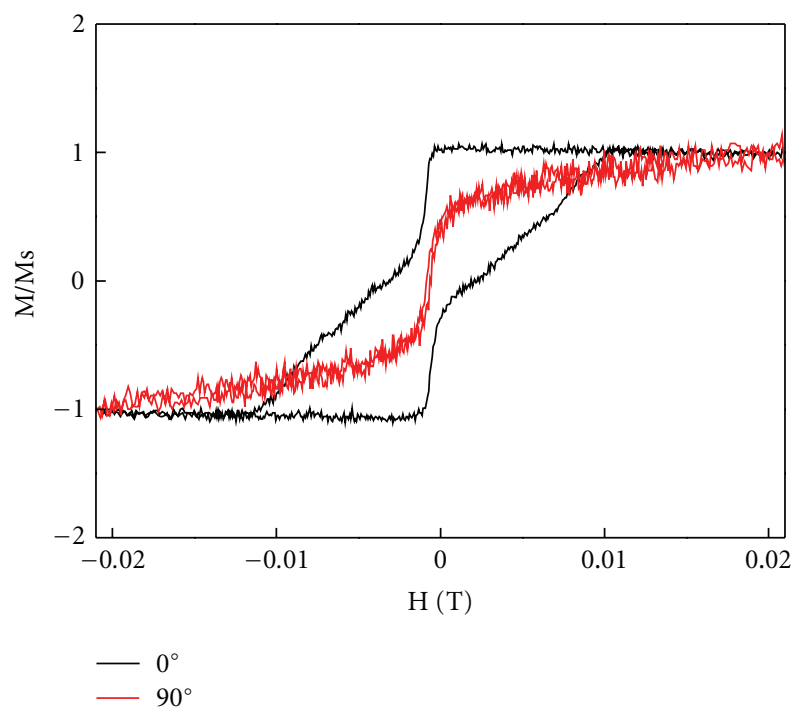

(b)

FIgURE 7: (a) Schematic drawing of the Stoner-Wohlfarth model for $\mathrm{S} / \mathrm{Py} / \mathrm{TbFe}_{2}$-coupled bilayers ( $\mathrm{H}$ : the driving magnetic field); (b) hysteresis loops of $\mathrm{S} / \mathrm{Py} / \mathrm{TbFe}_{2}$-coupled bilayers with field in plane $\left(\phi=0\right.$ and $\left.90^{\circ}\right)$.

\section{Acknowledgments}

The author gratefully acknowledges Professor Mikhail Indenbom, Mr. JAY Jean-Philippe, and Mr. BEN YOUSSEF Jamal for their guidance, supports, and useful discussions. This project is supported by K. C. Wong Education Foundation in Hong Kong and Opening Funding of National Key Laboratory of Science and Technology on Advanced Composites in Special Environments (no. HIT. KLOF.2009029) in Harbin Institute of Technology.

\section{References}

[1] A. E. Clark and H. S. Belson, "Giant room-temperature magnetostrictions in $\mathrm{TbFe}_{2}$ and $\mathrm{DyFe}_{2}$," Physical Review B, vol. 5, no. 9, pp. 3642-3644, 1972.

[2] Arthur E. Clark and Henry S. Belson, "Magnetostriction of terbium-iron and erbium-iron alloys," IEEE Transactions on Magnetics, vol. MAG-8, no. 3, pp. 477-479, 1972.

[3] P. J. Grundy, D. G. Lord, and P. I. Williams, "Magnetostriction in TbDyFe thin films," Journal of Applied Physics, vol. 76, no. 10, pp. 7003-7005, 1994.

[4] A. Speliotis, O. Kalogirou, and D. Niarchos, "Magnetostrictive properties of amorphous and partially crystalline TbDvFe thin films," Journal of Applied Physics, vol. 81, no. 8, pp. 5696-5698, 1997.
[5] H. Takagi, S. Tsunashima, S. Uchiyama, and T. Fujii, "Stress induced anisotropy in amorphous Gd-Fe and Tb-Fe sputtered films," Journal of Applied Physics, vol. 50, no. 3, pp. 1642-1644, 1979.

[6] E. Quandt, "Multitarget sputtering of high magnetostrictive Tb-Dy-Fe films," Journal of Applied Physics, vol. 75, no. 10, pp. 5653-5655, 1994.

[7] D. W. Forester, C. Vittoria, J. Schelleng, and P. Lubitz, "Magnetostriction of amorphous $\mathrm{Tb}_{x} \mathrm{Fe}_{1-x}$ thin films," Journal of Applied Physics, vol. 49, no. 3, pp. 1966-1968, 1978.

[8] J. Huang, C. Prados, J. E. Evetts, and A. Hernando, "Giant magnetostriction of amorphous $\mathrm{Tb}_{x} \mathrm{Fe}_{1-x}(0.10<\mathrm{x}<0.45)$ thin films and its correlation with perpendicular anisotropy," Physical Review B, vol. 51, no. 1, pp. 297-304, 1995.

[9] E. Quandt and A. Ludwig, "Giant magnetostrictive multilayers (invited)," Journal of Applied Physics, vol. 85, no. 8, pp. 62326237, 1999.

[10] E. Quandt, A. Ludwig, J. Betz, K. Mackay, and D. Givord, "Giant magnetostrictive spring magnet type multilayers," Journal of Applied Physics, vol. 81, no. 8, pp. 5420-5422, 1997.

[11] H. D. Chopra, M. R. Sullivan, A. Ludwig, and E. Quandt, "Magnetoelastic and magnetostatic interactions in exchangespring multilayers," Physical Review B, vol. 72, Article ID 054415, 7 pages, 2005.

[12] E. D. T. D. Lacheisserie, K. Mackay, J. Betz, and J. C. Peuzin, "From bulk to film magnetostrictive actuators," Journal of Alloys and Compounds, vol. 275-277, pp. 685-691, 1998.

[13] J. P. Jay, F. Petit, J. Ben Youssef, M. V. Indenbom, A. Thiaville, and J. Miltat, "Magnetostrictive hysteresis of $\mathrm{TbCo} / \mathrm{CoFe}$ multilayers and magnetic domains," Journal of Applied Physics, vol. 99, no. 9, Article ID 093910, 2006.

[14] J. B. Youssef, N. Tiercelin, F. Petit, H. Le Gall, V. Preobrazhensky, and P. Pernod, "Statics and dynamics in giant magnetostrictive $\mathrm{Tb}_{x} \mathrm{Fe}_{1-x}-\mathrm{Fe}_{0.6} \mathrm{Co}_{0.4}$ Multilayers for MEMS," IEEE Transactions on Magnetics, vol. 38, no. 5 I, pp. 2817-2819, 2002.

[15] G. Engdahl, Handbook of Giant Magnetostrictive Materials, chapter 6, AP, 2000.

[16] R. J. Gambino and J. J. Cuomo, "Selective resputtering-induced anisotropy in amorphous films," Journal of Vacuum Science and Technology, vol. 15, no. 2, pp. 296-301, 1978.

[17] Y. Suzuki, J. Haimovich, and T. Egami, "Bond-orientational anisotropy in metallic glasses observed by x-ray diffraction," Physical Review B, vol. 35, no. 5, pp. 2162-2168, 1987.

[18] D. Mergel, H. Heitmann, and P. Hansen, "Pseudocrystalline model of the magnetic anisotropy in amorphous rare-earth transition-metal thin films," Physical Review B, vol. 47, no. 2, pp. 882-891, 1993.

[19] G. Suran, M. Ouahmane, and R. Zuberek, "Correlations between the in-plane uniaxial anisotropy and magnetostriction in amorphous $\left(\mathrm{Co}_{93} \mathrm{Zr}_{7}\right)_{100-X}(\mathrm{RE})_{X}$ thin films," IEEE Transactions on Magnetics, vol. 30, no. 2 pt 2, pp. 723-725, 1994.

[20] T. Ambrose, R. L. Sommer, and C. L. Chien, "Angular dependence of exchange coupling in ferromagnet/antiferromagnet bilayers," Physical Review B, vol. 56, no. 1, pp. 83-86, 1997. 

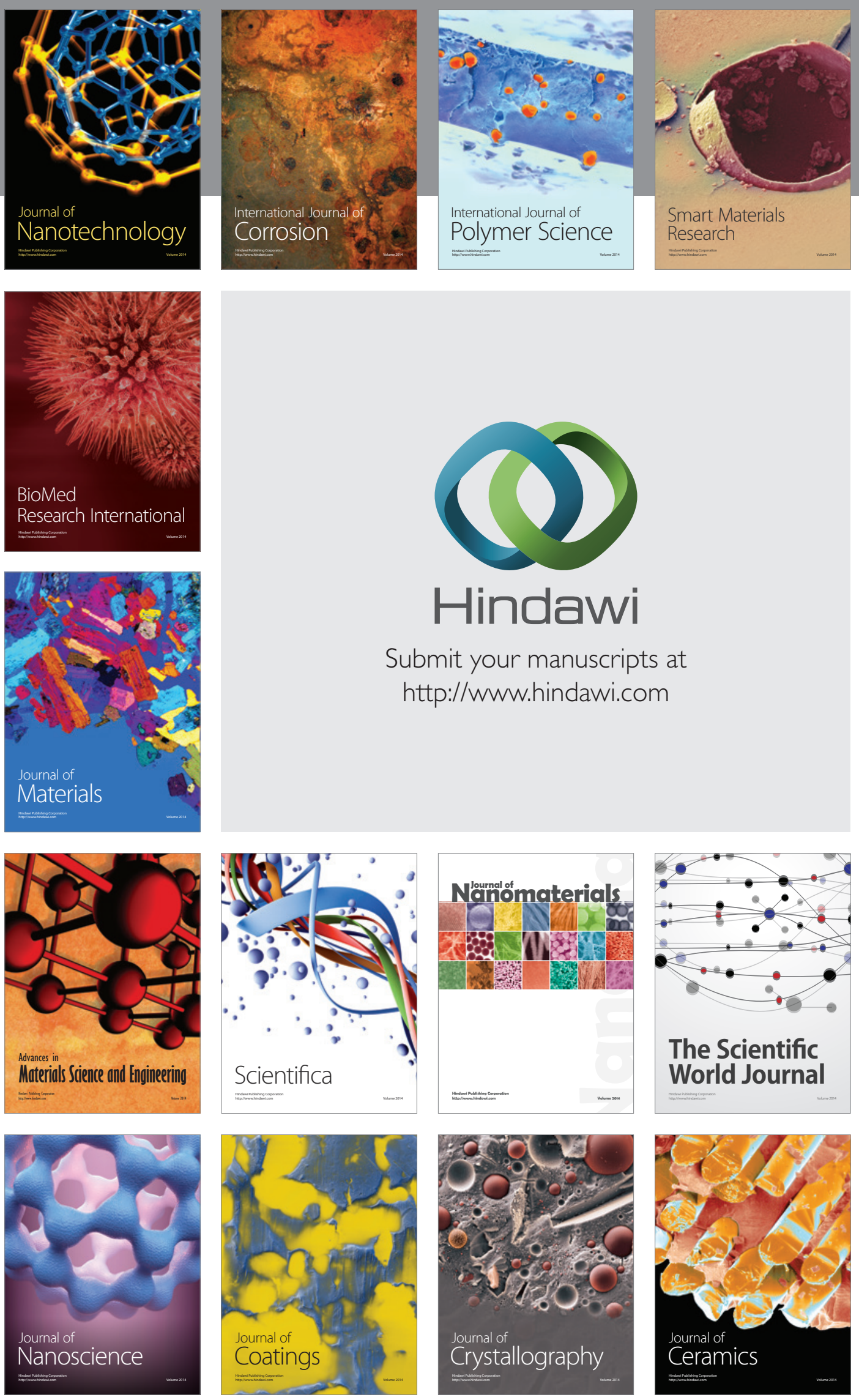

The Scientific World Journal

Submit your manuscripts at

http://www.hindawi.com

\section{World Journal}

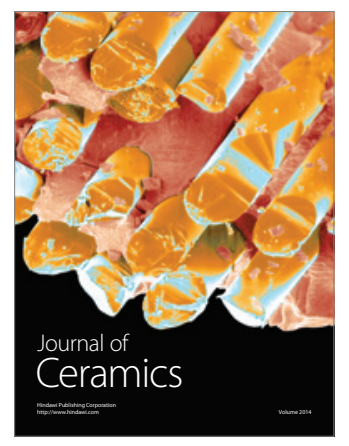

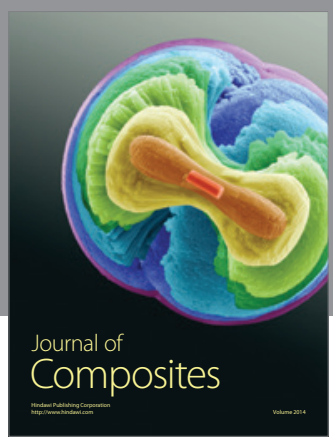
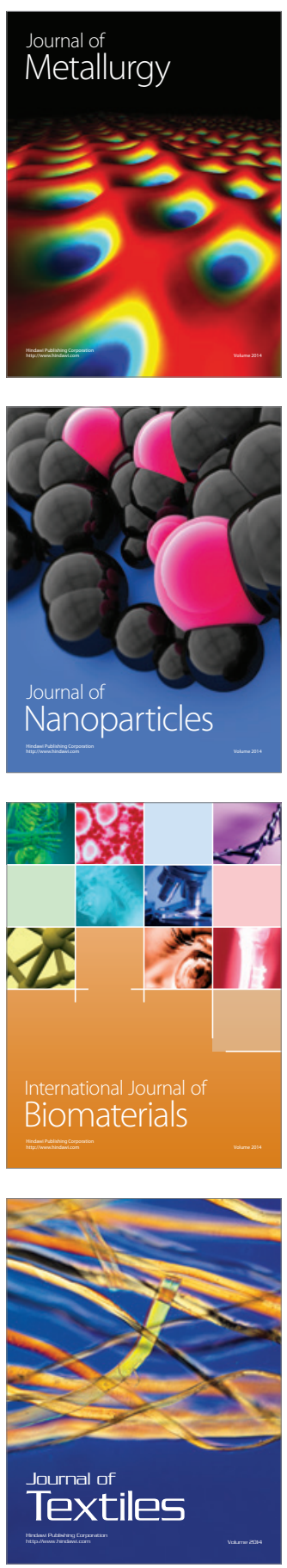